\title{
On Gravity and the Uncertainty Principle
}

\author{
Ronald J. Adler* \\ David I. Santiago ${ }^{\dagger}$ \\ Gravity Probe B, W.W.Hansen Experimental Physics Laboratory, and Department of Physics, Stanford University, Stanford, \\ Ca 94305-4085
}

(February 4, 2008)

\begin{abstract}
Heisenberg showed in the early days of quantum theory that the uncertainty principle follows as a direct consequence of the quantization of electromagnetic radiation in the form of photons. As we show here the gravitational interaction of the photon and the particle being observed modifies the uncertainty principle with an additional term. From the modified or gravitational uncertainty principle it follows that there is an absolute minimum uncertainty in the position of any particle, of order of the Planck length. A modified uncertainty relation of this form is a standard result of superstring theory, but the derivation given here is based on simpler and rather general considerations with either Newtonian gravitational theory or general relativity theory.
\end{abstract}

\section{INTRODUCTION}

Max Planck discovered the eponymous constant $\hbar$ when studying black body radiation in 1900 [1]. He realized immediately that the constants $\hbar$ and $c$ and $G$ determine a natural scale, now called the Planck scale, which is easily gotten by dimensional analysis [2]. The Planck distance, time, mass, and energy are

$$
\begin{array}{r}
L_{p} \equiv \sqrt{\frac{G \hbar}{c^{3}}} \simeq 1.6 \times 10^{-35} \mathrm{~m}, \quad T_{p} \equiv \frac{L_{p}}{c}=\sqrt{\frac{G \hbar}{c^{5}}} \simeq 0.54 \times 10^{-43} \mathrm{sec} \\
M_{p} \equiv \frac{\hbar}{c L_{p}}=\sqrt{\frac{\hbar c}{G}} \simeq 2.2 \times 10^{-8} \mathrm{~kg}, \quad E_{p} \equiv \sqrt{\frac{\hbar c^{5}}{G}} \simeq 2.0 \times 10^{9} \mathrm{~J}=1.2 \times 10^{19} \mathrm{GeV} .
\end{array}
$$

From its construction the Planck scale should be relevant when the system considered is quantum mechanical $(\hbar)$, involves high velocities and high energies $(c)$, and gravity is important $(G)$. One such system is the very early universe. Another is the collision of elementary particles such as quarks at about the Planck energy, but to achieve the Planck energy in a laboratory would probably require an accelerator about the size of a galaxy. Yet another system in which the Planck scale is relevant is the cloud of virtual particles surrounding any real particle, since the virtual particles may in principle have arbitrarily high energies.

Much work has gone into constructing a quantum theory of gravity appropriate to the Planck scale, but with little practical success. The only theory thus far that seems to be a plausible candidate is superstring theory [3].

Motivation for the present work originated at a talk by John Schwarz at the Stanford Linear Accelerator Center in 1996, in which he presented a modified uncertainty principle as a result of superstring theory and scale inversion symmetry. He asked if, in view of its simplicity, it might be more general than superstring theory or any particular quantum gravity theory, and perhaps derivable by simpler means. We hope this work partially answers that question.

We do not consider any specific quantum gravity theories here, but instead show that the Heisenberg uncertainty principle is modified when we combine quantum theory and some basic concepts of gravity. We give four separate derivations of the modified uncertainty principle: dimensional analysis in Newtonian theory, an approximate calculation in Newtonian theory, dimensional analysis in general relativity theory, and an approximate calculation in general relativity theory 断. All four derivations are heuristic and somewhat rough, as befits a discussion of the uncertainty principle. Moreover the derivations based on Newtonian theory should not be taken too seriously since the Newtonian theory is action-at-a-distance, which is certainly inappropriate for a photon moving at $c$; our purpose in including the Newtonian derivation is to show that the modified uncertainty principle appears to follow from rather general

*adler@relgyro.stanford.edu

†david@spacetime.stanford.edu 
considerations on gravity, in particular that the additional gravitational term is linear in the energy or momentum of the photon.

A minimum position uncertainty arises immediately from the modified uncertainty principle, of order of the Planck distance. One might consider this to be a minimum physically meaningful distance, and thereby question whether any theory based on a a smaller scale, eg. a spacetime continuum, really makes operational sense. Additionally, since all measurements dealing with small distances in particle physics are really large momentum scattering experiments, the very concept of a spacetime continuum is doubly suspect. Further speculations of this nature are contained in the conclusions.

\section{THE UNCERTAINTY PRINCIPLE A LA HEISENBERG}

Heisenberg in 1923 obtained the uncertainty principle on very general grounds, based only on general principles of optics and the quantization of electromagnetic radiation in the form of photons [5.1]. We recall his approach briefly. Consider a wave scattering from an electron into a microscope and thereby giving a measurement of the position of the electron. According to optics and intuition, with an electromagnetic wave of wavelength $\lambda$ we cannot obtain better precision than

$$
\Delta x_{H} \approx \lambda
$$

Such a wave is quantized in the form of photons, each with a momentum

$$
p=\frac{h}{\lambda}
$$

In order to interact with the electron an entire photon in the wave must scatter and thereby impart to the electron a significant part of its momentum, which produces an uncertainty in the electron momentum of about $\Delta p \approx p$. Thus we obtain the standard Heisenberg position-momentum uncertainty relation

$$
\Delta x_{H} \Delta p \approx \lambda\left(\frac{h}{\lambda}\right) \approx h \approx \hbar,
$$

No mention has been made here of the gravitational interaction between the photon and the electron, which we consider below.

\section{NEWTONIAN THEORY, DIMENSIONAL ESTIMATE}

We first estimate the effects of gravity in a very rough and heuristic way using Newtonian gravitational theory, with the assumption that the photon behaves as a classical particle with an effective mass equal to its energy divided by $c^{2}$ 肺. Suppose the electron is in an experimental region of characteristic size $L$, inside of which it interacts with the photon. It will experience an acceleration due to gravity,

$$
\ddot{\vec{r}}=-\frac{G\left(E / c^{2}\right)}{r^{2}} \hat{r}
$$

where $r$ is the distance between electron and photon. During the interaction, which occurs in characteristic time $L / c$, the electron will acquire, due to gravity, a velocity and move a distance, given respectively by

$$
\Delta v \approx \frac{G E}{c^{2} r^{2}}\left(\frac{L}{c}\right), \quad \Delta x_{G} \approx \frac{G E}{c^{2} r^{2}}\left(\frac{L}{c}\right)^{2} .
$$

These will be uncertain since the photon scatters electromagnetically from the electron at some indeterminate time during the interaction. The electron may be anywhere in the interaction region so the electron-photon distance should be of order $r \approx L$, which is the only distance scale in the problem. Since the photon energy is related to the momentum by $E=p c$ we may also express this as

$$
\Delta x_{G} \approx \frac{G p}{c^{3}}
$$


Noting that the electron momentum uncertainty must be of order of the photon momentum, and using the Planck length $L_{p}^{2} \equiv G \hbar / c^{3}$ as a parameter, we have

$$
\Delta x_{G} \approx \frac{G \Delta p}{c^{3}}=\left(\frac{G \hbar}{c^{3}}\right) \frac{\Delta p}{\hbar}=L_{p}^{2} \frac{\Delta p}{\hbar} .
$$

This is our main result. We add this uncertainty to the Heisenberg relation (3) to obtain the modified uncertainty relation

$$
\Delta x \approx \frac{\hbar}{\Delta p}+L_{p}^{2} \frac{\Delta p}{\hbar}
$$

We refer to this as the extended uncertainty principle - or more descriptively as the gravitational uncertainty principle (GUP). Note that it is invariant under

$$
\frac{\Delta p L_{p}}{\hbar} \longleftrightarrow \frac{\hbar}{\Delta p L_{p}}
$$

That is, it has a kind of momentum inversion symmetry [3].

\section{NEWTONIAN THEORY, APPROXIMATE CALCULATION}

We may also make a more explicit estimate than the above using Newtonian theory. As before we suppose the electron is in an experimental region of characteristic size $L$ and interacts with the photon as it crosses the region. The photon scatters electromagnetically from the electron at some uncertain time and at some uncertain position inside the experimental region. Consider first the transverse impulse, that is the motion imparted to the electron perpendicular to the photon direction. We take the photon direction to be $x$ and the transverse direction to be $y$ as in figure 2. The photon passes very rapidly so the electron moves very little in the time it takes the photon to cross the experimental region, and we may thus take $y \approx y_{0}$ that is we use an impulse approximation. The acceleration is then

$$
\ddot{y}=\frac{G\left(E / c^{2}\right)}{r^{2}}\left(\frac{y}{r}\right)=\frac{G(p / c)}{r^{2}}\left(\frac{y}{r}\right) \approx \frac{G(p / c) y_{0}}{\left(y_{0}^{2}+c^{2} t^{2}\right)^{3 / 2}} .
$$

We integrate this to get the transverse velocity impulse,

$$
\Delta \dot{y} \approx \frac{2 G p}{c^{2} y_{0}}\left(\frac{c T}{\sqrt{y_{0}^{2}+c^{2} T^{2}}}\right)
$$

where $T=L / c \geq y_{0}$ is the characteristic interaction time. We thus have roughly

$$
\Delta \dot{y} \approx \frac{2 G p}{c^{2} y_{0}} .
$$

Due to this gravitational velocity impulse there will be a change in the position of the electron, which is intrinsically uncertain, given approximately by $\Delta y \approx(\Delta \dot{y} / 2) / T$ or

$$
\Delta y_{G} \approx \frac{G p T}{c^{2} y_{0}} \approx \frac{G p}{c^{3}}
$$

where we have taken $y_{0}$ to be of order of but less than $L=c T$. This is the same result (3.3) as we obtained by somewhat more crude dimensional arguments in section III.

A similar analysis can be done for motion in the longitudinal direction, with the result

$$
\Delta x_{G} \approx \frac{G p}{c^{3}}\left[\ln \left(\frac{2 L}{x_{0}}\right)-1\right]
$$

where $x_{0}$ is the initial position of the electron. Since $x_{0}$ must be of order but less than $L=c T$ and the log is a very slowly varying function we obtain about the same result as (4.4) for the longitudinal uncertainty, 


$$
\Delta x_{G} \approx \frac{G p}{c^{3}} .
$$

One should be justifiably suspicious of the Newtonian derivations since Newtonian theory treats the gravitational field in front of the radiation as action-at-a-distance, whereas the gravitational field actually propagates at $c$ and cannot extend in front of the radiation. The nature of the gravitational field will become clear when we discuss the calculation using linearized general relativity.

\section{GENERAL RELATIVITY THEORY, DIMENSIONAL ESTIMATE}

The arguments in the preceding two sections are only marginally convincing as heuristic arguments since they are based on action-at-a-distance Newtonian gravitational theory, with the ad hoc assumption that the energy of the photon produces a gravitational field. In this section and the following we give a dimensional estimate and an approximate calculation based on general relativity theory, free of such drawbacks [6,2, th.

The field equations of general relativity are

$$
G_{\mu \nu}=-\left(\frac{8 \pi G}{c^{4}}\right) T_{\mu \nu} .
$$

The left side has the units of inverse distance squared, since it is constructed from second derivatives and squares of first derivatives of the metric. Thus on dimensional grounds we may write the left hand side in terms of deviations of the metric from flat, in schematic order of magnitude dimensional form, as

$$
L H S \approx \frac{\delta g_{\mu \nu}}{L^{2}},
$$

where $\delta g_{\mu \nu}$ denotes the deviation of the metric from Lorentzian, and $L$ is the same characteristic size as used in sec. III. Similarly the energy-momentum tensor has the units of an energy density, so its components must be roughly equal to the photon energy over $L^{3}$. Thus we can write the right side of the field equations schematically as

$$
R H S \approx\left(\frac{8 \pi G}{c^{4}}\right) \frac{E}{L^{3}} \approx \frac{G p}{c^{3} L^{3}} .
$$

Equating the dimensional estimates in (5.2) and (5.3) we get an estimate for the deviation of the metric,

$$
\delta g_{\mu \nu} \approx \frac{G p}{c^{3} L} .
$$

This deviation corresponds to a fractional uncertainty in all positions in the region $L$, which we identify with a fractional uncertainty in position, $\Delta x_{G} / L$. Thus we have an uncertainty in position due to the gravitational interaction given by

$$
\frac{\Delta x_{G}}{L} \approx \delta g_{\mu \nu} \approx \frac{G p}{c^{3} L}, \quad \Delta x_{G} \approx \frac{G p}{c^{3}} .
$$

As should be expected the characteristic size $L$ has canceled out of the relation. Finally the uncertainty in momentum of the electron must be comparable to the photon momentum, $\Delta p \approx p$, and we obtain the same relation (3.4) as before for $\Delta x_{G}$.

\section{GENERAL RELATIVITY THEORY, APPROXIMATE CALCULATION}

To make an approximate calculation we use linearized general relativity theory. From the general energy momentum tensor of the electromagnetic field it is easy to show that for radiation moving in the $x$ direction the specific form of the energy- momentum tensor is [7,6]

$$
T_{m u \nu}=F_{\mu \alpha} F_{\nu}^{\alpha}+\frac{1}{4} F_{\alpha \beta} F^{\alpha \beta}=\rho\left(\begin{array}{rrrr}
1 & -1 & 0 & 0 \\
-1 & 1 & 0 & 0 \\
0 & 0 & 0 & 0 \\
0 & 0 & 0 & 0
\end{array}\right),
$$


where $\rho=\left(E^{2}+B^{2}\right) / 2$ is the energy density of the radiation field, and may be a function of $x-c t, y$, and $z$, corresponding to a truncated plane wave. The equations of linearized general relativity theory follow from (5.1), with the metric taken to be Lorentz plus a small perturbation, $g_{\mu \nu}=\eta_{\mu \nu}+h_{\mu \nu}$. They are

$$
\begin{gathered}
\square\left[h_{\mu \nu}-\frac{1}{2} \eta_{\mu \nu} h\right]=-\left(\frac{8 \pi G}{c^{4}}\right) T_{\mu \nu}, \quad h \equiv \eta^{\mu \nu} h_{\mu \nu}=h_{\alpha}^{\alpha}, \\
\square=\frac{\partial^{2}}{\partial(c t)^{2}}-\frac{\partial^{2}}{\partial x^{2}}-\frac{\partial^{2}}{\partial y^{2}}-\frac{\partial^{2}}{\partial z^{2}},
\end{gathered}
$$$$
\text { Lorentz gauge condition }\left[h_{\mu}^{\nu}-\frac{1}{2} \eta_{\mu}^{\nu} h\right]_{\mid \nu}=0 \text {. }
$$

It is straight-forward to solve this system with the energy momentum tensor given in (6.1). We are interested only in the inhomogeneous solution, and the system then reduces to a form involving only one unknown function,

$$
h_{\mu \nu}=f(x-c t, y, z)\left(\begin{array}{rrrr}
1 & -1 & 0 & 0 \\
-1 & 1 & 0 & 0 \\
0 & 0 & 0 & 0 \\
0 & 0 & 0 & 0
\end{array}\right), \quad \square f=-\left(\frac{8 \pi G}{c^{4}}\right) \rho .
$$

For convenience we choose the energy density to be a product,

$$
\rho(x-c t, y, z)=\rho_{\|}(x-c t) \rho_{\perp}(y, z),
$$

and it then follows that the metric function $f$ is also a product of the form $f(x-c t, y, z)=f_{\|}(x-c t) f_{\perp}(y, z)$, with

$$
\left(\frac{\partial^{2}}{\partial y^{2}}+\frac{\partial^{2}}{\partial z^{2}}\right) f_{\perp}(y, z)=\left(\frac{8 \pi G}{c^{4}}\right) \rho_{\perp}(y, z),, \quad f_{\|}(x-c t)=\rho_{\|}(x-c t) .
$$

Until now the energy density function has been arbitrary. We now choose a specific function which is convenient for our purposes. For this we take a cylinder as the envelope of the radiation, inside of which the energy density oscillates at twice the frequency of the radiation field; the oscillations however may be ignored since we are interested in an average uncertainty in position. Thus we take the energy density to be a constant $\rho_{0}$ inside a cylinder of length $L$ and radius $R$, with $R$ and $L$ comparable in magnitude. Then the solution to (6.5), in cylindrical coordinates $x-c t, r, \varphi$, is

$$
\begin{gathered}
f=\frac{4 G\left(E / c^{2}\right)}{L} g(r) \theta_{L}(x-c t)=\frac{4 G p}{c^{3} L} g(r) \theta_{L}(x-c t), \\
g(r)=\left\{\begin{array}{ll}
r^{2} / R^{2}, & r<R \\
1+\ln \left(r^{2} / R^{2}\right), & r>R
\end{array}\right\}, \quad \theta_{L}(x-c t) \equiv \theta(x-c t) \theta(c t-x-L)
\end{gathered}
$$

Here $E=\pi R^{2} L \rho_{0}$ is the total energy of the radiation, and $\theta_{L}$ is the "double" theta function defined above - which is equal to 0 ahead of and behind the radiation cylinder. Notice that the gravitational field does not extend ahead of the radiation field, unlike in the Newtonian theory. Notice also that the apparent logarithmic divergence of the external field is not a problem since physical effects involve the derivative of $f$ which falls off like $1 / r$. According to (6.3) and (6.6) the metric takes the rather elegant form

$$
d s^{2}=c^{2} d t^{2}-\left(d x^{2}+d y^{2}+d z^{2}\right)+f(c d t-d x)^{2}
$$

It is clear that since $g(r)$ is of order 1 inside and near the cylinder of radiation the deviation of the metric from Lorentz is there of order $4 G p / c^{3} L$ and we thereby obtain, using the same idea as in section $\mathrm{V}$,

$$
\frac{\Delta x_{G}}{L} \approx \frac{G p}{c^{3} L} \approx \frac{G \Delta p}{c^{3} L} \quad x_{G} \approx \frac{4 G \Delta p}{c^{3}} .
$$


We may also estimate the gravitationally induced motion of the electron using the above metric and the geodesic equation of motion, to obtain an alternative derivation of the position uncertainty. The geodesic equation of motion is

$$
\frac{d^{2} x^{i}}{d s^{2}}+\left\{\begin{array}{c}
i \\
\alpha \beta
\end{array}\right\} \frac{d x^{\alpha}}{d s} \frac{d x^{\beta}}{d s}=0, \quad \alpha, \beta=0,1,2,3, \quad i=1,2,3 .
$$

For an electron moving reasonably slowly the line element is about $d s=c d t$ and the $\alpha=\beta=0$ term dominates, so the equations of motion are the usual Newtonian limit equations

$$
\frac{d^{2} x^{i}}{d t^{2}}=-\left\{\begin{array}{c}
i \\
00
\end{array}\right\} c^{2}
$$

From the metric (6.7) the Christoffel symbols are easily found and we obtain for motion in the longitudinal $x$ and transverse $r$ directions

$$
\frac{d^{2} x}{d t^{2}}=\frac{1}{2} \frac{\partial f}{\partial x} c^{2}, \quad \frac{d^{2} r}{d t^{2}}=-\frac{1}{2} \frac{\partial f}{\partial r} c^{2}
$$

where we assume motion in the $x$ and $r$ direction.

Longitudinal motion is easy to analyze and rather informative. The derivative of $f$ gives two delta functions. As the front of the radiation cylinder passes the electron it first gives the electron a velocity impulse of

$$
\Delta \dot{x}=\frac{2 G p}{c^{2} L} g(r)
$$

and then as the back of the radiation cylinder passes the electron receives an equal and opposite velocity impulse and stops. In the time of passage it has moved

$$
\Delta x_{G}=\frac{2 G p}{c^{2} L} g(r) T=\frac{2 G p}{c^{3}} g(r) \approx \frac{2 G p}{c^{3}},
$$

since $g(r)$ is of order 1 in and near the radiation cylinder. Thus we obtain the same result (3.4) as previously. Notice that the gravitational field of the radiation only acts as it passes over the electron, not before and not after.

For the transverse motion we differentiate $f$ with respect to $r$ to find

$$
\frac{d^{2} r}{d t^{2}}=-\frac{4 G p}{c^{2} L}\left\{\begin{array}{ll}
r / R^{2}, & r<R \\
1 / r, & r>R
\end{array}\right\} \theta_{L}(x-c t)
$$

so that in the region of the cylinder we have very roughly a velocity impulse and a corresponding position change $\Delta r \approx(\Delta \dot{r} / 2) T$, given by

$$
\Delta \dot{r}=\frac{4 G p}{c^{2} L R} T, \quad \Delta r=\frac{2 G p}{c^{2} L R} T^{2} \approx \frac{2 G p}{c^{3}}
$$

That is once again we find that the transverse motion corresponding to an uncertainty in position due to gravity is the same as in (3.3) or (3.4).

\section{THE MINIMAL DISTANCE UNCERTAINTY}

The GUP has a remarkable consequence. If the photon momentum and $\Delta p$ are chosen to be very small then the electron position is imprecise because the long photon wavelength gives poor resolution. If the photon momentum and $\Delta p$ are chosen to be very large, then the gravitational field of the photon makes the electron position very imprecise. Between the two extremes there is a minimum position uncertainty, which we find from (3.5) to be

$$
\Delta x_{\min } \approx 2 \sqrt{\frac{G \hbar}{c^{3}}}=2 L_{p}, \text { for } \Delta p \approx \sqrt{\frac{\hbar c^{3}}{G}} \frac{E_{p}}{c} .
$$

This means that we can never localize the position of a particle such as an electron to better than about the Planck distance.

Similar analyses of spacetime using the path integral formalism lead to analogous conclusions; spacetime at small distances and times undergoes quantum fluctuations, and at the Planck scale the fluctuations are of the same order as the distances involved [2,8]. 


\section{CONCLUSIONS}

We have shown, using Newtonian and general relativistic gravity, that the position-momentum uncertainty principle of quantum mechanics is modified by an additional term. In both theories it is clear that the extra term must be proportional to the energy or momentum of the photon, so on purely dimensional grounds the order of magnitude of the extra term is uniquely determined. As a consequence there is an absolute minimum uncertainty in the position of any particle such as an electron. Not surprisingly the minimum is of order of the Planck distance.

In view of the absolute minimum position uncertainty one may plausibly question whether any theory based on shorter distances, such as a spacetime continuum, really makes sense. Indeed in light of the fact that laboratory experiments which probe small distance properties of particles are all high energy scattering experiments, one might conclude that spacetime at such small scales may not be a useful concept, and that spacetime at the Planck scale may not even exist in any meaningful operational sense. Such ideas are not new and were espoused in the era of S-matrix theory in the 1960s, that is that the scattering amplitude expressed $n$ terms of input and output momenta may be the fundamental reality of high energy physics, and not point-like or string-like particles in a spacetime continuum [9]. One might even speculate that the spacetime continuum concept actually impedes physics in the same way that the concept of an ether impeded physics in the 19th century. As such, a theoretical structure based entirely on momenta, such as a modern version of S-matrix theory, might be desirable and interesting.

\section{ACKNOWLEDGEMENTS}

This work was supported by NASA grant NAS 8-39225 to Gravity Probe B. John Schwarz posed the question studied here in a talk at the Stanford Linear Accelerator Center in fall 1996, but is of course not responsible for our interpretation of it. Finally the Gravity Probe B theory group at Stanford provided many stimulating discussions.

[1] J. H. Weaver, ed. The World of Physics, Simon and Schuster, New York, 1987. Volume II section N contains the Nobel prize award address of Planck in 1918. and Heisenberg in 1932.

[2] C. W. Misner, K. S. Thorne, and J. A. Wheeler, Gravitation, W. H. Freeman and Co. San Francisco, 1973. The Planck scale is discussed in sec. 1.2 and linearized general relativity theory in section 18.1.

[3] M. B. Green, J. H. Schwarz, and E. Witten, Superstring Theory, Cambridge University Press, 1987.

[4] H. C. Ohanian and R. Ruffini, Gravitation and Spacetime, 2nd ed., W. W. Norton and Co., New York, 1994. Chapter 1 contains Newtonian gravitationaltheory, and chapter 3 contains the linearized approximation of general relativity.

[5] L. I. Schiff, Quantum Mechanics, 3rd ed., McGraw Hill, New York, 1968. See chapter 1, section 4, on quantum measurement.

[6] R. J. Adler, M. Bazin, and M. M. Schiffer, Introduction to General Relativity, 2nd ed., McGraw Hill, New York, 1975. We use these notations and sign conventions. The energy momentum tensor of the electromagnetic field is in chapter 10 .

[7] R. C. Tolman, Relativity, Thermodynamics and Cosmology, Clarendon Press, Oxford, 1934. See chapter 8. Tolman does an analysis of the photons gravitational field that is similar to that done here, but less general and for an essentially point photon.

[8] G. Amelino-Camelia, Nature, 398, p216, 1999.

[9] G. Chew, S-Matrix Theory of Strong interactions, W. A. Benjamin Inc., New York, 1961. 